\title{
SCIDiC
}

\author{
International Journal of Dentistry and Oral Science (IJDOS) \\ ISSN: 2377-8075
}

\section{Clinical Practice Guidelines For Management Of Oral Submucous Fibrosis}

Research Article

Dhanvanth.M ${ }^{1}$, Uma Maheswari.T.N2*

'Saveetha Dental College and Hospitals, Saveetha Institute of Medical and Technical Sciences (SIMATS), Saveetha University, Chennai, India.

${ }^{2}$ Head of Admin, Department of Oral Medicine and Radiology, Saveetha Dental College and Hospitals, Saveetha Institute of Medical and Technical Sciences (SIMATS), Saveetha University, Chennai, India.

Abstract

Background: Oral sub mucous fibrosis (OSMF) is a chronic, progressive, debilitating, scarring and crippling disorder of the oral cavity. It is characterized by inflammation, increased deposition of sub mucosal collagen and formation of fibrotic bands in the oral tissues, which increasingly limitmouth opening. Many OSMF treatment protocols have been proposed to alleviate the signs and symptoms of the disorder and there is overwhelming evidence that as areca nut is primary cause, stopping its use may have a considerable effect on symptoms rather than reversing pre-existing fibrosis. Even though several treatment modalities have been recommended, only a few are evidence based and can be considered for the optimal management of oral sub mucous fibrosis. The objective of this study was to propose and review treatment protocol to be followed for the management of oral sub mucous fibrosis. We reviewed several evidence-based studies and through this review we recommend topical interventions as the first-line of treatment since they are associated with low risk of systemic side effects.

Conclusion: The effectiveness of the interventions cannot be reliably assessed. Larger, better conducted trials of longer duration are required to properly effect treatments for oral sub mucous fibrosis.Due to limitations in the number of evidencebased studies, larger evidence-based clinical trialsliterature reviews are needed to further improve the guideline protocol for the effective management of oral sub mucous fibrosis.

Keywords: Management; Oral Sub Mucous Fibrosis; Areca Nut; Evidence-Based Treatment; Conservative Therapy; Surgical Therapy.

\section{Introduction}

Oral sub mucous fibrosis (OSMF) is an oral precancerous condition characterized by inflammation and progressive fibrosis of the sub mucosal tissues resulting in marked rigidity and trismus. [1] Oral sub mucous fibrosis (OSMF) has significant potential for malignant transformation. Oral mucosa is a unique tissue, lined by keratinized and non-keratinized stratified squamous epithelium and underlying connective tissue (lamina propria).[2] The oral mucosa is continuously exposed to chemicals, microorganisms, thermal changes and mechanical irritants (tobacco, areca nut, alcohol, etc). The epithelial and connective tissue components of the oral mucosa demonstrate acute and chronic reactive changes in response to the above stressors. The keratinized epithelium which is present on the dorsum of the tongue, such as hard palate and attached gingiva, shows less reactive changes to the stressors as compared to the non-keratinized epithelium, which is seen everywhere in the oral cavity including the buccal mucosa, labial mucosa, alveolar mucosa and specialized mucosa.[3, 4]. Oral submucous fibrosis (OSMF) is a chronic, insidious, progressive, debilitating, scarring, irreversible, complex and crippling disorder of the oral cavity. $[5,6]$

The rapid increase in the prevalence of this disease is due to an upsurge in the popularity of commercially available areca nut and tobacco preparations - gutkha, pan masala, flavored areca nut, mawa, etc., in Asian countries. [7] It causes significant morbidity, in terms of loss of mouth function as tissues become rigid and mouth opening becomes difficult, and mortality because of transformation into squamous cell carcinoma.[8]

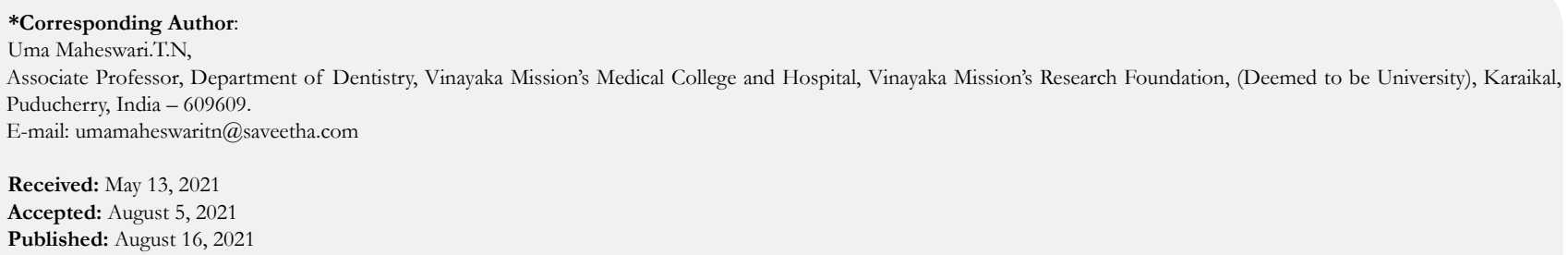

Copyright: Uma Maheswari.T.N ${ }^{\top} 2021$. This is an open-access article distributed under the terms of the Creative Commons Attribution License, which permits unrestricted use, distribution and reproduction in any medium, provided the original author and source are credited. 
The fundamental goals of treatment are to shorten the duration of limited mouth opening, provide relief from pain \& increase disease-free periods while the secondary goals would be to lessen the fibrous bands $\&$ severity of recurrences. The aim of this study was to review and propose a treatment protocol to be followed for the management of oral sub mucous fibrosis. [9]

\section{Aetiology}

The aetiology of OSMF is obscure, although various hypotheses are proposed, suggesting multifactorial origins, such as chewing of areca nut and its flavored formulations (most common), chronic nutritional deficiencies (especially iron, Vitamin B complex and protein) and genetic predisposition, autoimmunity Excessive use of areca nut and its flavored formulations disrupts the haemostatic equilibrium between synthesis and degeneration. The copper ion in areca nut increases the activity of lysyl oxidase leading to unregulated collagen production, thereby causing oral fibrosis. This leads to the production of free radicals and reactive oxygen species, which are responsible for high rate of oxidationperoxidation of polyunsaturated fatty acids. $[10,11]$

OSMF is a disease of middle age group with peak incidence observed in the second to fourth decade of life. The sex distribution of OSMF varies geographically. The most common oral site for OSMF is buccal mucosa and retromolar region, followed by soft palate, faucial pillars, floor of mouth, tongue, labial mucosa and gingiva.[12]

\section{Predisposing Factors}

Areca nut (betel nut) chewing is one of the most common causes of OSMF which contains tannins and alkaloids. Arecoline is the main agent and active metabolite in fibroblast stimulation and proliferation, thereby inducing collagen synthesis. Deficiency of iron (anemia), Vitamin B complex, minerals, and malnutrition are promoting factors that leads to deranged healing and resultant scarring and fibrosis $[13,14]$. The increase in CD4 cells and cells with HLA-DR in these diseased tissues shows activation of most lymphocytes and increased number of Langerhans cells.

\section{Clinical Presentation}

The oral sub mucous fibrosis appears causes different effects on varies stages in oral cavity. In early stage, it clinically represents with burning sensation, stomatitis, blister formation, blanching of oral mucosa, presence of thin palpable fibrous bands. In moderate stage along with burning sensation, stomatitis, gradual decrease in mouth opening, xerostomia, loss of taste sensation, difficulty in blowing cheeks, rigid oral mucosa, blanching of buccal mucosa, labial mucosa, soft palate, floor of mouth, tongue, thick palpable fibrous bands and shrunken uvula. In advanced stage, along with moderate stage presentation it appears to be loss of suppleness of mucosa, restriction of tongue movement, mottled white or opaque or white marble like appearance of oral mucosa, de-papillation of tongue and involvement of pharyngeal and esophageal mucosa.[15]

OSMF is insidious in origin and does not regress, either spontaneously or with cessation of habit. The disease remains either stationary or becomes severe, leaving an individual handicapped, both physically and psychologically.[16] Usually, the OSMF lesion shall be biopsied, especially if there are ulcerative, nodular, erythematous and suspicious areas. The histologically proven severe or moderate epithelial dysplasia shall be treated in the lines of management of carcinoma. Non dysplastic or mildly dysplastic cases must be kept under long-term observation and shall be advised antioxidant therapy after discontinuation of habit.[17]

\section{Pathogenesis}

Pathogenesis is believed to involve juxta-epithelial inflammatory reaction and fibrosis in the oral mucosa, probably due to increased cross-linking of collagen through up-regulation of lysyl oxidase activity. Fibrosis, or the build-up of collagen, results from the effects of areca nut, which increases collagen production (e.g., stimulated by arecoline, an alkaloid) and decreases collagen degradation. Thus, Oral submucous fibrosis is now considered a collagen metabolic disorder.[18]

\section{Diagnosis}

The diagnosis for OSMF is clinical and histological. Clinically, one or more of the following symptoms should be present:Blanching of oral mucosa defined as a persistent, white, marble-like appearance of the oral mucosa, which may be localized, diffuse or reticular, tough, leathery texture of the mucosa and palpable, whitish, fibrous bands. On histopathological diagnosis OSMF is characterized by epithelial atrophy with loss of rete ridges and hyalinization of the lamina propria and the underlying muscle. The initial pathology of OSMF is characterized by mixed inflammation and edema, and large fibroblasts. $[19,20]$ Later, collagen bundles with early hyalinization are seen, and the inflammatory infiltrate contains lymphocytes and plasma cells. In advance stages OSMF is characterized by formation of thick bands of collagen and hyalinization extending into the submucosal tissues and decreased vascularity. [21]

\section{Management}

The management for OSMF can be divided into

- Conservative therapy

- Surgical therapy

Conservative therapy:

o Physical therapy

Physical exercise regimen

Splints or other mouth opening devices

Microwave diathermy

o Medical therapy

$>$ Steroids

$>$ Interferon c

$>$ Immunized milk

Placental extracts

$>$ Levamisole

$>$ Colchicine 
Figure 1. Management for Oral sub mucous fibrosis.

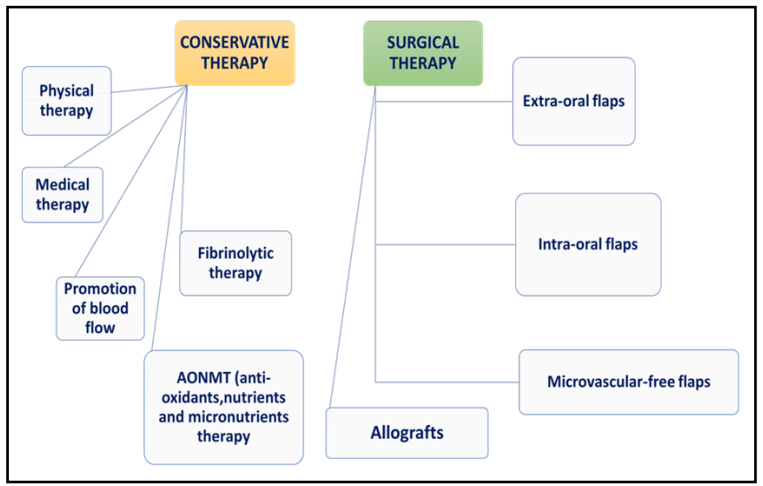

o Promotion of blood flow

Pentoxyphylline

Buflomedil hydrochloride

Nylidrin

o Anti-oxidants, Nutrients and Micro-nutrients therapy

B-carotene

Lycopene

Tea pigments

$>$ Vitamins

$>$ Mineral supplements

o Fibrinolytic therapy

Hyaluronidase

$>$ Chymotrypsin

$>$ Collagenase

Surgical therapy:

o Extra-oral flaps

Split thickness skin graft

Superficial temporal fascia pedicled flap

Temporalis pedicled flap

$>$ Nasolabial flap

Platysmamyocutaneous muscle flap

o Intra oral flaps

Tongue flap

Palatal island flap

Buccal pad of fat

o Micro vascular-free flaps

Radial forearm free flap

Anterolateral thigh flap

o Allografts

Collagen membrane

Alloderm

\section{Conclusion}

Despite years of research, no single treatment modality has proved to be effective for OSMF.Overwhelming evidence that areca nut is primary cause, stopping its use may have a considerable effect on managing the condition, Targeted education and legislative changes for reducing areca nut dependence still remain an essential part of the management of OSMF globally. Clinically diagnosed oral sub mucous fibrosis should undergo histopathological examination to detect the presence of epithelial dysplasia for arriving at the correct treatment plan. By categorizing the patients by risk factors based on clinical factors such as location, size and histopathological features with evidence of dysplasia, specific management for individuals need to be devised based on risk status. The effectiveness of the interventions cannot be reliably assessed. Larger, better conducted trials of longer duration are required to properly effect treatments for oral sub mucous fibrosis.

\section{References}

[1]. Arakeri G, Brennan PA. Oral submucous fibrosis: an overview of the aetiology, pathogenesis, classification, and principles of management. $\mathrm{Br} \mathrm{J}$ Oral Maxillofac Surg. 2013 Oct 1;51(7):587-93

[2]. International Agency for Research on Cancer. Betel-quid and areca nut chewing and some areca nut derived nitrosoamines, vol. 85. Lyon:IARC; 2004. p. 123-129.

[3]. Kerr AR, Warnakulasuriya S, Mighell AJ, Dietrich T, Nasser M, Rimal J, et al. A systematic review of medical interventions for oral submucous fibrosis and future research opportunities. Oral Dis. 2011 Apr;17 Suppl 1:42-57. Pubmed PMID: 21382138.

[4]. Chole RH, Gondivkar SM, Gadbail AR, Balsaraf S, Chaudhary S, Dhore $\mathrm{SV}$, et al. Review of drug treatment of oral submucous fibrosis. Oral Oncol. 2012 May 1;48(5):393-8.

[5]. Fedorowicz Z, Shih-Yen EC, Dorri M, Nasser M, Newton T, Shi L. Interventions for the management of oral submucous fibrosis. Cochrane Database Syst. Rev. 2008(4);CD007156

[6]. Haque MF, Meghji S, Nazir R, Harris M. Interferon gamma (IFN- $\gamma$ ) may reverse oral submucous fibrosis. J Oral Pathol Med. 2001 Jan;30(1):12-21.

[7]. Khanna JN, Andrade NN. Oral submucous fibrosis: a new concept in surgical management. Report of 100 cases. Int J Oral Maxillofac Surg. 1995 Dec;24(6):433-9.Pubmed PMID: 8636640.

[8]. Patil PG, Parkhedkar RD. New graft-stabilizing clip as a treatment adjunct for oral submucous fibrosis. J Prosthet Dent. 2009 Sep;102(3):191-2.Pubmed PMID: 19703627.

[9]. Cox S, Zoellner H. Physiotherapeutic treatment improves oral opening in oral submucous fibrosis. J. Oral Pathol. Med. 2009 Feb;38(2):220-6.

[10]. Gupta DS, Gupta MK, Golhar BL. Oral submucous fibrosis-clinical study and management by physiofibrolysis (MWD). J Indian Dent Assoc. 1980;52(1):375-8.

[11]. Warnakulasuriya S, Kerr AR. Oral submucous fibrosis: a review of the current management and possible directions for novel therapies. Oral Surg Oral Med Oral Pathol Oral Radiol. 2016 Aug;122(2):232-41.Pubmed PMID: 27422422.

[12]. Chen Z, Chen HB, Huang WX, Huang Q. The clinical effect of micro- 
wave radiation in treating oral mucous membrane diseases. J Clin Stomatol. 2006;22:750.

[13]. Rajalalitha P, Vali S. Molecular pathogenesis of oral submucous fibrosis--a collagen metabolic disorder. J Oral Pathol Med. 2005 Jul;34(6):321-8.Pubmed PMID: 15946178.

[14]. Bhatnagar P, Rai S, Bhatnagar G, Kaur M, Goel S, Prabhat M. Prevalence study of oral mucosal lesions, mucosal variants, and treatment required for patients reporting to a dental school in North India: In accordance with WHO guidelines. J Family Community Med. 2013 Jan;20(1):41-8.Pubmed PMID: 23723730

[15]. More C, Shah P, Rao N, Pawar R. Oral submucous fibrosis: an overview with evidence-based management. Int J Oral Health Sci Adv. 2015;3(3):40-9.

[16]. Markiewicz MR, Margarone III JE, Barbagli G, Scannapieco FA. Oral mucosa harvest: an overview of anatomic and biologic considerations. EurAssocUrol. 2007 Oct 1;5(5):179-87.

[17]. van der Waal I. Potentially malignant disorders of the oral and oropharyngeal mucosa; terminology, classification and present concepts of management. Oral Oncol. 2009 Apr-May;45(4-5):317-23.Pubmed PMID: 18674954.

[18]. More CB, Gupta S, Joshi J, Varma SN. Classification system for oral submucous fibrosis. J Indian Acad Oral Med Radiol. 2012;24(1):24-9.

[19]. Maher R, Sankaranarayanan R, Johnson NW, Warnakulasuriya KA. Evaluation of inter-incisor distance as an objective criterion of the severity of oral submucous fibrosis in Karachi, Pakistan. Eur J Cancer B Oral Oncol. 1996 Sep;32B(5):362-4.Pubmed PMID: 8944843.

[20]. Jontell M, Holmstrup H. Red and white lesions of the oral mucosa. In: Greenberg M, Glick M, Ship J, editors. Burket's Oral Medicine. 11th ed. Jericho, England: Hamilton; 2008. p. 88-9. Back to cited text no. 7

[21]. Daftary D, Murti P, Bhonsle R, Gupta P, Mehta F, Pindborg J. Oral precancerous lesions and conditions of tropical interest. In: Prabhu S, Wilson D, Daftary D, Johnson N, editors. Oral Diseases in the Tropics. Jericho, England: Oxford University Press; 1993. p. 417-22. 